\title{
Genome instability model of metastatic neuroblastoma tumorigenesis by a dictionary learning algorithm
}

\author{
Salvatore Masecchia ${ }^{1 \dagger}$, Simona Coco $^{2 \dagger}$, Annalisa Barla ${ }^{1}$, Alessandro Verri ${ }^{1}$ and Gian Paolo Tonini ${ }^{3 *}$
}

\begin{abstract}
Background: Metastatic neuroblastoma (NB) occurs in pediatric patients as stage $4 \mathrm{~S}$ or stage 4 and it is characterized by heterogeneous clinical behavior associated with diverse genotypes. Tumors of stage 4 contain several structural copy number aberrations (CNAs) rarely found in stage 4S. To date, the NB tumorigenesis is not still elucidated, although it is evident that genomic instability plays a critical role in the genesis of the tumor. Here we propose a mathematical approach to decipher genomic data and we provide a new model of NB metastatic tumorigenesis.
\end{abstract}

Method: We elucidate NB tumorigenesis using Enhanced Fused Lasso Latent Feature Model (E-FLLat) modeling the array comparative chromosome hybridization $(\mathrm{aCGH})$ data of 190 metastatic NBs (63 stage 4S and 127 stage 4). This model for aCGH segmentation, based on the minimization of functional dictionary learning (DL), combines several penalties tailored to the specificities of aCGH data. In DL, the original signal is approximated by a linear weighted combination of atoms: the elements of the learned dictionary.

Results: The hierarchical structures for stage $4 \mathrm{~S}$ shows at the first level of the oncogenetic tree several whole chromosome gains except to the unbalanced gains of 17q, 2p and 2q. Conversely, the high CNA complexity found in stage 4 tumors, requires two different trees. Both stage 4 oncogenetic trees are marked diverged, up to five sublevels and the 17q gain is the most common event at the first level (2/3 nodes). Moreover the 11q deletion, one of the major unfavorable marker of disease progression, occurs before $3 p$ loss indicating that critical chromosome aberrations appear at early stages of tumorigenesis. Finally, we also observed a significant $(p=0.025)$ association between patient age and chromosome loss in stage 4 cases.

Conclusion: These results led us to propose a genome instability progressive model in which NB cells initiate with a DNA synthesis uncoupled from cell division, that leads to stage $4 S$ tumors, primarily characterized by numerical aberrations, or stage 4 tumors with high levels of genome instability resulting in complex chromosome rearrangements associated with high tumor aggressiveness and rapid disease progression.

\section{Background}

Neuroblastoma (NB) is a clinically and biologically heterogeneous pediatric cancer, the onset of which can be localized or disseminated disease. Disseminated tumors are classified as clinical stages $4 \mathrm{~S}$ and 4 . Stage $4 \mathrm{~S}$ occurs in infants, usually have a good prognosis without any

\footnotetext{
* Correspondence: gp.tonini@irpcds.org

${ }^{\dagger}$ Equal contributors

${ }^{3}$ Neuroblastoma Laboratory, Onco/Hematology Laboratory, Department of Woman and Child Health, University of Padua, Pediatric Research Institute, Fondazione Città della Speranza, Padua, Corso Stati Uniti, 4, 35127 Padua, Italy

Full list of author information is available at the end of the article
}

treatments, although a small fraction of stage $4 \mathrm{~S}$ patients can have a disease progression requiring chemotherapy [1]. Both infants and children can present stage $4 \mathrm{NB}$, but older patients have usually a worse outcome with a rapid disease progression that leads to death in more than half of patients [2].

Genome-wide studies have showed that gains of chromosomes 2 p, 7 and $17 q$ are frequently present together with losses of $1 p, 4 p, 9 p, 11 q$, and 14q in stage 4 tumors, whereas stage $4 \mathrm{~S}$ tumors frequently display numerical copy number aberrations (CNAs) [3-5]. The origin of such complex chromosomal aberrations is still unclear, 
and there are currently no accurate models of NB tumorigenesis. Nonetheless, experimental evidence indicates that NB is characterized by a high level of genome instability [6] and that CNAs accumulate in an agedependent manner [7, 8].

As a result of the publicly available high-throughput array comparative genomic hybridization $(\mathrm{aCGH})$ data repositories in the Gene Expression Omnibus (GEO), it is now possible to investigate CNAs in large cohorts of NB samples. A signal measured with the aCGH technology is made of a piecewise constant component plus some composite noise. The typical analysis on such data is segmentation, which is the automatic detection of chromosome loci where CNAs (amplifications or deletions) occur, as shown in the Fig. 1. Beyond that, it is crucial to understand how these alterations co-occur. This turns to identifying shared patterns (latent features) in the data, which may reveal a genotype-phenotype relationship.

Several methods have been suggested for the extraction of CNAs based on different principles, such as filtering (or smoothing), segmentation, breakpoint-detection and calling [9-16], taking into account one sample at a time [17].

Especially in cancer diseases, where mutations happen very frequently, joint-analysis of aCGH samples could be helpful to filter out unshared mutations among (at least a subset of) samples. One of the first works applying this approach was performed by Pique-Regi et al. [18] where the authors extended their previous model [13] to the "multi-sample" analysis. Following this scheme, many other approaches were proposed usually extending the "one-by-one" criterion to a "multi-sample" application [19-21] and in some cases extending this approach also to the joint-normalization of data [22].

Moreover, some interesting recent results were obtained by adopting statistical learning methods based on regularization for a joint segmentation of many aCGH profiles at once. Previous results [11, 23-25] obtained following this method are based on total variation $(T V)$ or fused lasso signal approximation.

In this context, we use E-FLLat (Enhanced Fused Lasso Latent Feature Model) [26], a novel model for aCGH segmentation, based on the minimization of functional dictionary learning (DL) combining several penalties tailored to the specificities of the data at hand. In $\mathrm{DL}$, the original signal (i.e., the aCGH sample) is approximated by a linear weighted combination of the atoms (i.e., a set of elementary alterations), which are the elements of a learned dictionary.

We assumed that each sample can be approximated by a weighted combination of some of the identified atoms. A simple example of this concept is shown in the Fig. 2 where the signal can be obtained as the weighted sum of three elementary alterations (atoms). In the analysis of the NB data, we first identified the atoms using E-FLLat; then, we applied an inference method [27] to place the atoms in a set of hierarchical structures (trees) that may shed light on NB oncogenesis.

Thus, we used the aCGH data and a statistical inference method based on dictionary learning to propose a Genome Instability Progressive (GIP) model of tumorigenesis

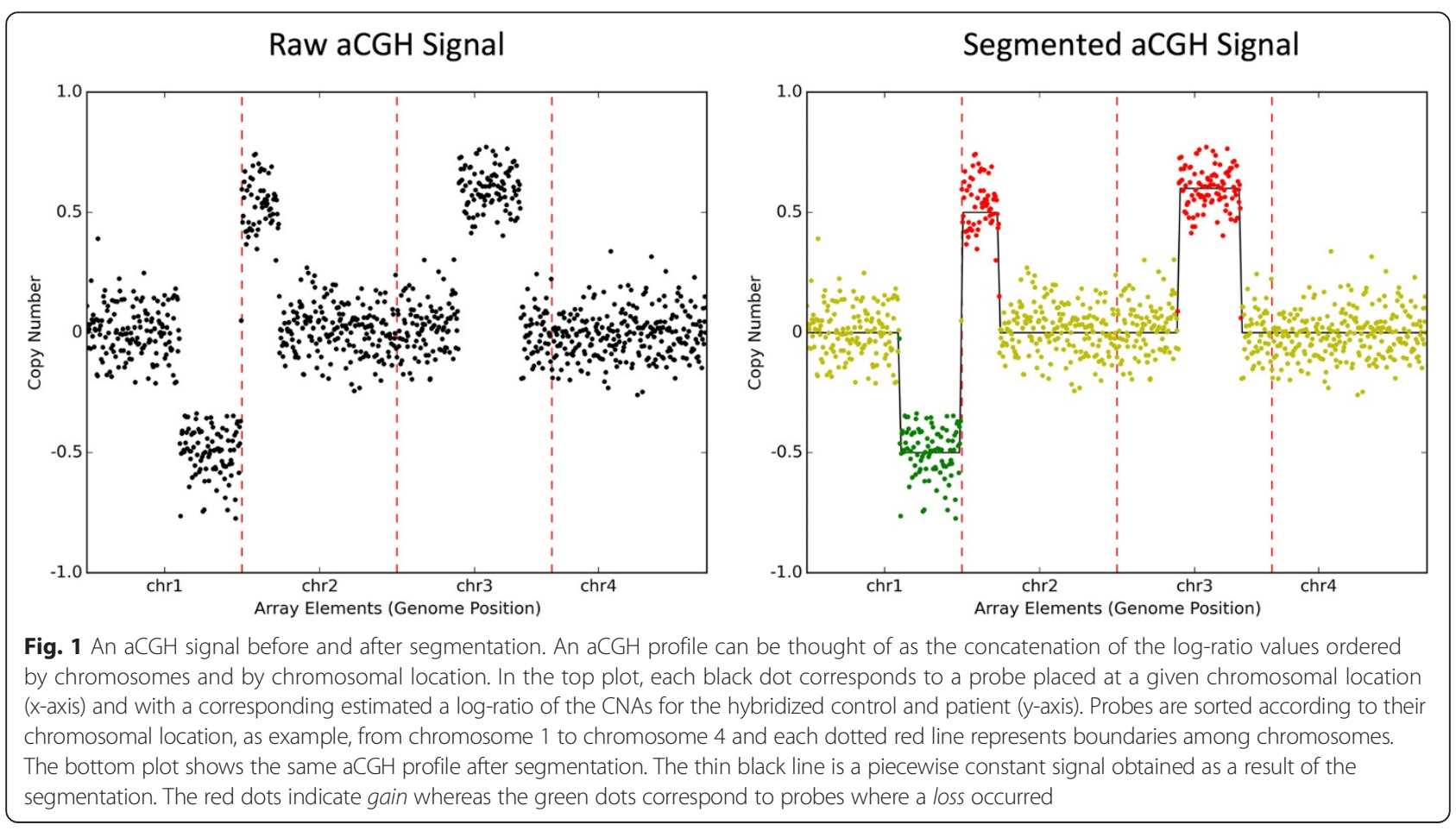




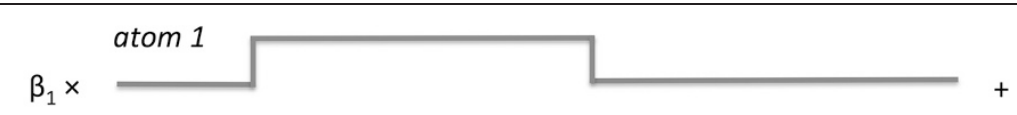

atom 2

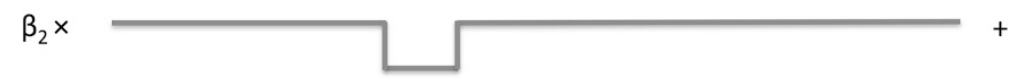

atom 3

$\beta_{3} \times \square \square$

Fig. 2 A piecewise constant signal as weighted linear combination of atoms. The piecewise constant signal at the bottom of the figure can be obtained by linearly combining a set of three elementary alterations (atoms). Each atom is multiplied by a weighting factor (coefficient) $\beta$ and then added up to obtain the final signal

for metastatic NB, which has one of the most aggressive known pediatric cancer phenotypes.

\section{Methods}

\section{Data description}

We analyzed six publicly available datasets from the GEO [28] for E-FLLat analysis (Table 1). We used a variation of the alignment algorithm of [29] consisting of three steps: 1) mapping to the reference genome, 2) signal smoothing for noise reduction, and 3) alignment.

\section{Data alignment and normalization}

1. Mapping of the Human Genome (HG19): First, we proceeded by mapping the probe sets on the HG19 [30]. The Agilent samples were mapped using mapping files from UCSC (44k and $105 k)^{1}$, whereas the Nimblegen data were mapped using the lift-over function available at $\mathrm{UCSC}^{2}$.

2. Normalization: For the Agilent platforms, we first performed a check of the quality control (QC) results, discarding those probes associated with a poor QC value. For the normalization of all of the data, we used CGHnormaliter [31]. Each input file (sample) has a corresponding normalized output containing information on the call, segmentation and normalized log-ratio of all 22 autosomes. As the output, the algorithm provides the CNAs estimated for each unique probe set on the chip.

3. Alignment: As opposed to Jong et al. [29], who sampled each chromosome $\mathrm{N}$ times, we decided to sample the chromosomal bands, excluding the non-coding short arms 13p, 14p, 15p and 22p and the sexual chromosomes. Each of the resulting 795 chromosomal bands was sampled $N=10$ times. For each of the new virtual 7950 probes, we performed a $\mathrm{K}-\mathrm{NN}$ procedure that assigns an estimated expression to the new virtual probe by considering $\mathrm{K}=10$ nearest neighbors all belonging to the same chromosomal band. $\mathrm{K}=10$ was the upper bound; therefore, we used ten values at most to estimate the assigned expression. Finally, also in contrast to Jong et al. [29], we excluded

Table 1 Description of aCGH data collected from public available datasets

\begin{tabular}{|c|c|c|c|c|c|}
\hline aPlatform & ${ }^{\mathrm{b}} \mathrm{GEO}$ code & ${ }^{\mathrm{c}} \mathrm{GEO}$ serie & ${ }^{\mathrm{d}} \mathrm{NB}$ stage $4 \mathrm{~S}$ & ${ }^{\mathrm{d}} \mathrm{NB}$ stage 4 & ${ }^{\mathrm{e}}$ Total samples \\
\hline AgilentCgh2×105k & GPL4093 & GSE25771 & 16 & 7 & 23 \\
\hline AgilentCgh4x44k & GPL2873 & GSE25771 GSE35953 & 10 & 39 & 49 \\
\hline AgilentCgh4x44k & GPL2879 & GSE25771 & 5 & 1 & 6 \\
\hline AgilentCgh4x44k & GPL5477 & GSE14109 GSE25771 GSE35953 & 17 & 73 & 90 \\
\hline AgilentCgh4x44k & GPL11633 & GSE26494 & 1 & 0 & 1 \\
\hline Nimblegen $4 \times 72 \mathrm{k}$ & GPL8971 & GSE26494 & 14 & 7 & 21 \\
\hline 'Total Samples & & & 63 & 127 & 190 \\
\hline
\end{tabular}

aPlatform type, ${ }^{\mathrm{b}} \mathrm{GPL}$ file code from GEO, ${ }^{\mathrm{C}} \mathrm{GEO}$ series number, ${ }^{\mathrm{d}}$ number of Stage 4 and $4 \mathrm{~S}$ neuroblastoma samples, ${ }^{\mathrm{e}}$ samples number used in the analysis 
the z-score transformation, due to its drastic effect on the signal mean. After the alignment and normalization, the dataset was composed of 190 samples represented by 7950 probes. The chromosomal bands contained 10 probes each.

\section{E-FLLat: a dictionary learning-based approach for aCGH segmentation}

E-FLLat is a dictionary learning-based model for aCGH segmentation [23]. We are given $S$ samples $\left(\mathrm{y}_{S}\right)_{1 \leq S \leq S}$, with $y_{s} \in \mathbb{R}^{L}$. The aim of dictionary learning is to seek $J$ simple atoms $\left(\boldsymbol{\beta}_{j}\right)_{1 \leq j \leq J}$ with $\beta_{j} \in \mathbb{R}^{L}$, which may provide a complete representation of all of the samples in the sense that

$$
y_{s} \cong \sum_{j=1}^{J} \theta_{j s} \beta_{j} \quad \forall s=1, \ldots, S
$$

for some vectors of coefficients $\boldsymbol{\theta}_{\boldsymbol{s}}=\left(\boldsymbol{\theta}_{\boldsymbol{j} \boldsymbol{s}}\right)_{1<j<J}$

Thus, the E-FLLat model is as follows:

$$
\begin{aligned}
& \min _{\boldsymbol{\theta}_{s}, \boldsymbol{\beta}_{j}} \sum_{\boldsymbol{s}=1}^{\boldsymbol{S}}\left\|\boldsymbol{y}_{\boldsymbol{s}}-\sum_{\boldsymbol{j}=1}^{\boldsymbol{J}} \boldsymbol{\theta}_{\boldsymbol{j}} \boldsymbol{\beta}_{\boldsymbol{j}}\right\|^{2}+\lambda \sum_{j=1}^{\boldsymbol{J}}\left\|\boldsymbol{\beta}_{\boldsymbol{j}}\right\|_{1}^{2} \\
& \quad+\boldsymbol{\mu} \sum_{\boldsymbol{j}=1}^{J} \boldsymbol{T} \boldsymbol{V}_{\boldsymbol{w}}\left(\boldsymbol{\beta}_{\boldsymbol{j}}\right)+\boldsymbol{\tau} \sum_{\boldsymbol{s}=1}^{S}\left\|\boldsymbol{\theta}_{\boldsymbol{s}}\right\|_{1}^{2} \\
& \text { s.t. } 0 \leq \boldsymbol{\theta}_{\boldsymbol{j} s} \leq \boldsymbol{\theta}_{\max } \quad \forall \boldsymbol{j}=1, \ldots, \boldsymbol{J} \forall \boldsymbol{s}=1, \ldots, \boldsymbol{S}
\end{aligned}
$$

The weighted total variation $T V_{w}\left(\beta_{j}\right)=\sum_{l=1}^{L-1} w_{l}$ $\left|\boldsymbol{\beta}_{\boldsymbol{l}+1, j}-\boldsymbol{\beta}_{l, j}\right|$ is a generalized total variation due to the presence of the weights $\boldsymbol{w}=\left(w_{l}\right)_{1 \leq l \leq L-1} \in \mathbb{R}^{L-1}$. This modification is introduced to relax the constraint of small jumps on the atoms at some points. In fact, we imposed $w_{l}=0$ at the boundaries between the chromosomes and at the chromosome centromeres. Elsewhere, $w_{l}$ was set according to a position-dependent weighting schema as in [32].

\section{Post-processing and dictionary interpretation}

After the segmentation process, the dictionary was postprocessed to set a level of detail that was sufficiently general for the subsequent step of investigation. If one probe was detected as altered by E-FLLat, the smallest chromosomal band that contains that probe was considered as altered. Then, alterations occurring on adjacent chromosomal bands were merged and considered as one alteration occurring on the merged band.

\section{Carcinogenesis tree reconstruction}

Once the E-FLLat approach identified the atoms, we used MTreeMix [27], a software package for learning and using mixture models of oncogenic trees, to describe evolutionary processes that are characterized by the ordered accumulation of permanent genetic changes.
A tree is a hierarchical structure with one root node and a well-ordered set of nodes. The elements composing the tree are nodes and links. The depth of a node is the distance in links from the root node. The $n$-th level is defined as the set of nodes with distance $n$ from the root node.

\section{Results}

E-FLLat provides a new representation of the data in terms of a set of atoms (dictionary) and matrix of coefficients $\Theta$. Each sample can be approximated by a sparse linear combination of the atoms weighted by its corresponding set of coefficients (columns of the $\Theta$ matrix). Each atom is a unique element of the learned dictionary and represents an elementary pattern of highly correlated alterations that co-occur in the dataset. We separately applied E-FLLat to the stage $4 \mathrm{~S}$ and 4 subsets, represented by two matrices (63×7950-dimensional and 127x7950-dimensional, respectively).

The atoms for stage $4 \mathrm{~S}$ and 4 are listed in Table 2. Each atom is the set of relevant CNAs selected by E-FLLat and post-processed as described above. The number of atoms was chosen according to a principal component analysis (PCA) analysis (see Additional file 1 Figure S1) and was applied separately for stage $4 \mathrm{~S}$ and stage 4 data matrices. The PCA showed that $90 \%$ of the covariance of stage $4 \mathrm{~S}$ samples can be explained using $J=19$ atoms, whereas $J=$ 42 atoms are required for stage 4 samples. Therefore, we chose $J=16$, which is sufficient to explain at least $70 \%$ of the data covariance in both cases.

Table 2 Atoms characterizing Stage $4 S$ and stage 4 samples

\begin{tabular}{lll}
\hline & Stage 4s & Stage 4 \\
\hline Atom & Chromosome bands & Chromosome bands \\
A1 & $+2,+12$ & $+2 p 22.3,+2 p 23$ \\
A2 & $+7,+17$ & $+17 q$ \\
A3 & $+6,+13$ & $+2 p 24.3,+7 q,+17 q$ \\
A4 & $-21 q,-4 p$ & $+2 p 24,-1 p$ \\
A5 & $-14 q$ & $+2 p 2,+12 q$ \\
A6 & $-8 p$ & $+17 p,+17 q 1$ \\
A7 & $+2 p 2,+7$ & +18 \\
A8 & $-14 q 2$ & $+2 p$ \\
A9 & $-14 q$ & $-3 p$ \\
A10 & $+2 p 1,+2 q,+7$ & $+17 p$ \\
A11 & $-4,-10$ & +7 \\
A12 & - & +12 \\
A13 & -3 & $+2 p 2$ \\
A14 & - & - \\
A15 & +2 & $-11 q$ \\
A16 & $+12,+17 q$ & - \\
\hline
\end{tabular}

${ }^{a}$ Atom 12 is not associated with any relevant alteration for stage $4 S$, A14 for both 4 S and 4, A16 for stage 4 
Figure 3 shows the representation coefficients $(\Theta$ matrix) for stage $4 S$ and stage 4 samples, whose atoms are listed in Table 2. As expected, the atoms identified by E-FLLat highlight that stage $4 \mathrm{~S}$ samples are characterized by numerical alterations (A1, A2, A3, A11, A13, A15), whereas the stage 4 data mainly show segmental aberrations. The only numerical alterations in stage 4 tumors affected chromosomes 18 and 7 (atoms A7 and A11).
After the identification of the relevant atoms, the trees were inferred using the MTreeMix algorithm. Each root node in the trees is associated with the portion of patterns described by the corresponding tree.

The hierarchical structures for stage $4 S$ and 4 tumors are depicted in Fig. 4. The stage $4 S$ tree shows six events (atoms) with probabilities that range from 0.81 to 0.99 , suggesting that they occur in most of the represented samples. As expected at the first level the atoms are

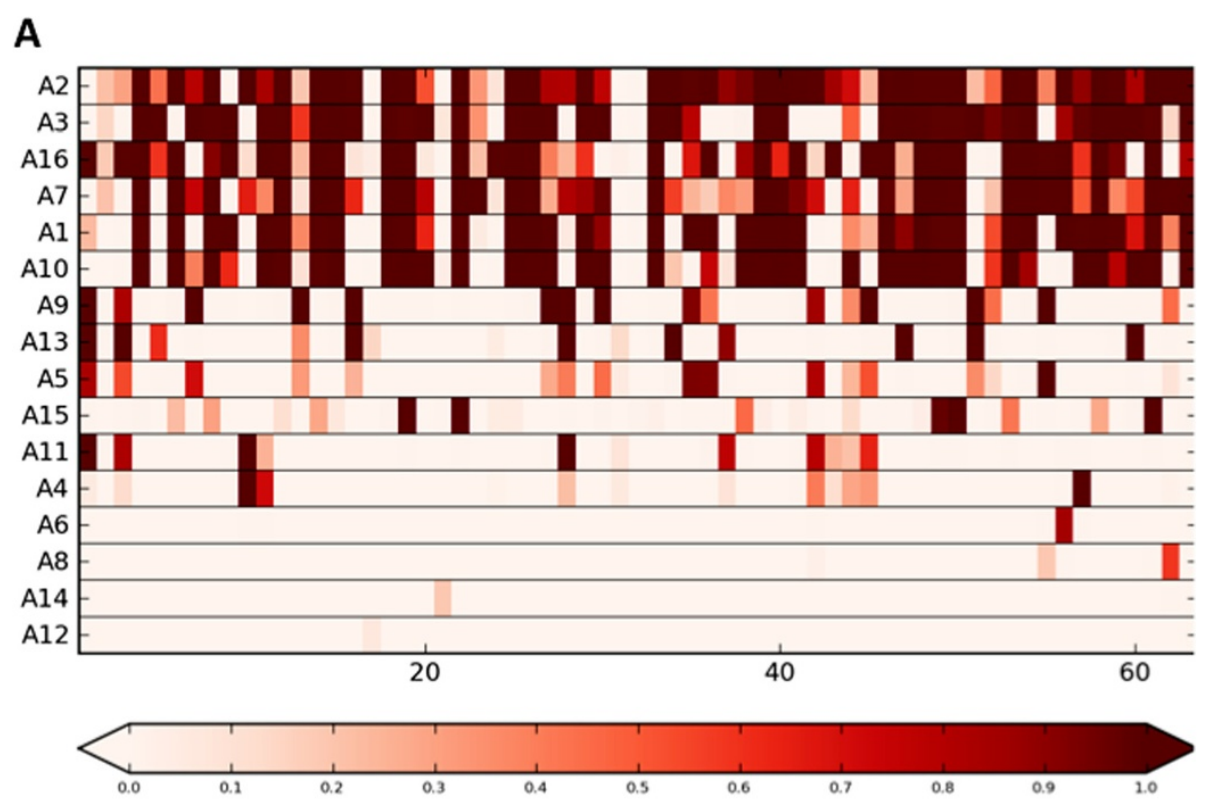

B

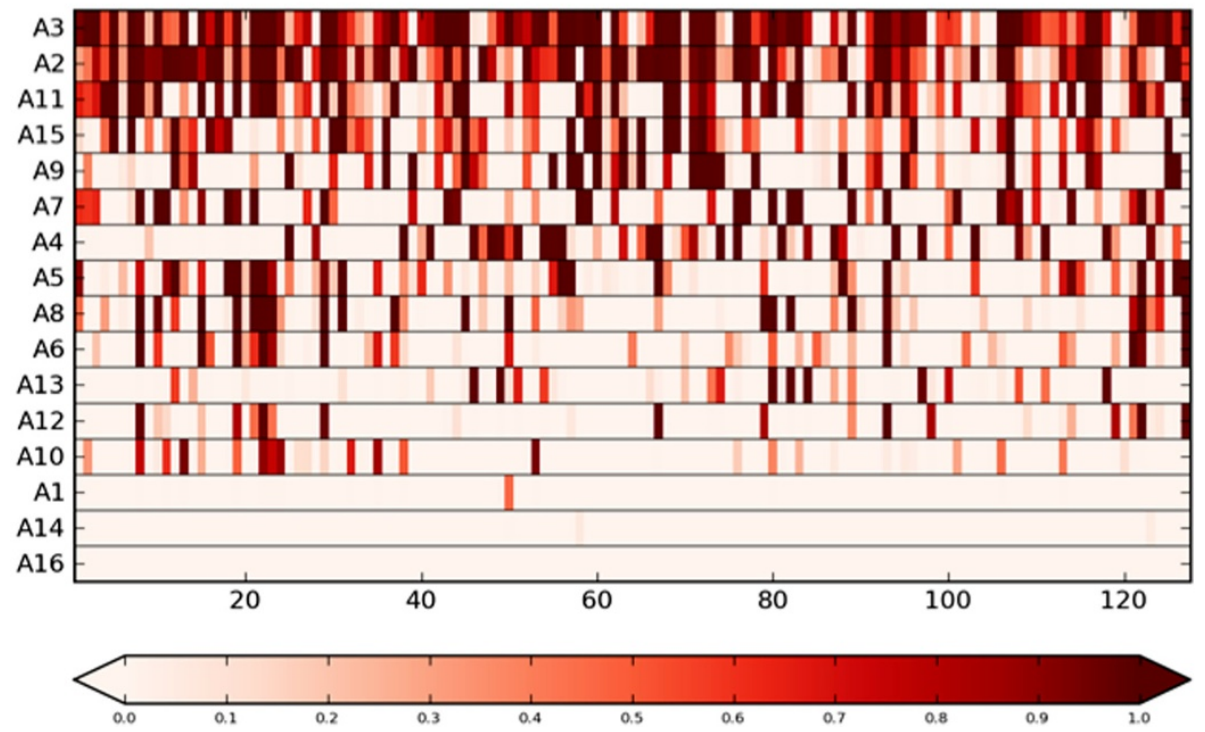

Fig. 3 Representation of $\Theta$ coefficients for stage $4 S$ and stage 4 tumors. The stage 4 S (a) and stage 4 (b) tumors are reported in the columns, whereas the atoms are in the rows. Each sample is approximated by a linear combination of atoms weighted by the $\Theta$ coefficients. The atoms in the $\Theta$ matrix are sorted according to their use in the sample representation, i.e., the most used atoms are in the top rows. The coefficients range from 0 to 1, as indicated by the underlying color bar, and darker hues correspond to higher coefficient values 

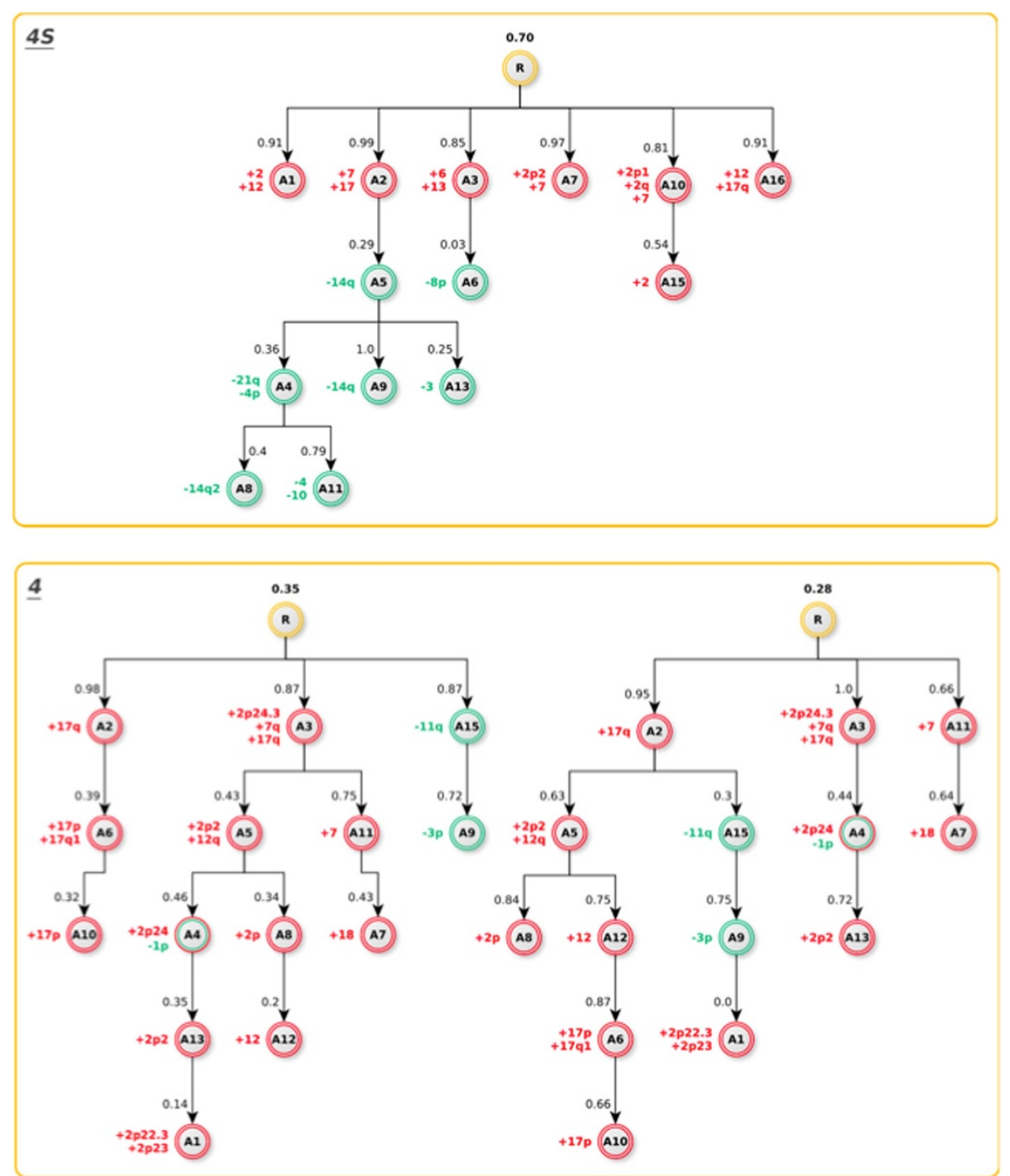

Fig. 4 Oncogenetic Trees. The reconstructed atom tree for stage 4S (top) shows several initial events of which only one with sublevels. Conversely, both stage 4 reconstructed atom trees (bottom) are marked branched (up to five sublevels) The root node R (yellow) is associated with a weight corresponding to the portion of mutation patterns represented by the tree. The missing portion is associated with the random mutations tree (data not shown). The edges are weighted according to the frequency of the corresponding mutation occurrence. Node color codes: the red node is associated with chromosome gain, the green node is associated with chromosome loss, and the green and red node indicates co-occurring loss and gain

characterized mainly by whole chromosome gains except to the unbalanced gains at $17 \mathrm{q}$ and $2 \mathrm{p}$ or $2 \mathrm{q}$.

Conversely, the high CNA complexity found in stage 4 tumors, requires two different trees to be properly represented. Notably each tree shows three atoms only at the first level, where the unbalance gain of $17 \mathrm{q}$ is the most common aberration occurring in two out of three nodes.

\section{Discussion}

Metastatic stage $4 \mathrm{~S}$ and stage $4 \mathrm{NB}$ tumors are characterized by distinct genome profiles and clinical behavior. In particular, the majority of stage $4 \mathrm{NB}$ display several structural CNAs that confer marked aggressiveness to these tumors [5]. How these chromosome aberrations are originated and how lead to transformation and carcinogenesis is still unclear, although experimental evidence indicates that genomic instability can play a critical role in the genesis of this tumor [7, 8].

Here, we propose the GIP model of carcinogenesis for metastatic NBs. To create this model, we used E-FLLat, a dictionary learning-based method that naturally groups the most relevant alterations in elementary patterns and sorts them according to how many times such patterns occurred in the data. This behavior is a built-in property of dictionary learning approaches, and in addition to being an advantage per se by providing a compact way of 
analyzing the coexisting alterations; it also eases the computational burden of the subsequent tree inference process. We used three different penalties that allowed us to segment the signal incorporating the characteristics of the aCGH data. Indeed, an aCGH signal is a stepwise constant, and alterations may co-occur in different chromosomes at the same time. We describe the EFLLat model, modifying some notations and illustrating the rationale behind the chosen constraints. When dealing with aCGH, $T V_{w}$ allows the treatment of signals generated by several chromosomes as a whole while still guaranteeing an independent analysis for each chromosome, ensuring the capability of identifying concomitant alterations occurring on different chromosomes. In the E-FLLat model, coefficients are constrained to be positive and are bounded by $\theta_{\max }=1$. This constraint prevents a cancellation effect in the representation of the signal, leading to a simpler matrix of coefficients and a matrix of atoms, which more clearly reveal the latent patterns in the data. In this way, the interpretability of the results is improved. For example, when losses and gains occur within data at the same locus, the model selects different atoms to describe them as different phenomena. The coefficients are further penalized by the term $\boldsymbol{\tau} \sum_{s=1}^{S}\left\|\boldsymbol{\theta}_{\boldsymbol{s}}\right\|_{1}^{2}$, which induces sparsity in the set of weights associated with each sample separately. This feature permitted the model to regulate how many different atoms of each sample can be combined to reconstruct the original signal. Then, we used the term $\sum_{j=1}^{J}\|\boldsymbol{\beta} \boldsymbol{j}\|_{1}^{2}$, which induces a structured sparsity in the columns of the matrix of the atoms. Only inexact algorithms can solve the minimization of the model in the E-FLLat function. In our implementation, we use an alternating proximal algorithm [26], which provides an approximation of the exact solution with a controlled error. The choice of the regularization parameters $(\lambda, \mu, \tau)$ is determined according to the Bayesian Information Criterion [33] that mitigates the problem of over-fitting by introducing a penalty term for the complexity of the model. The choice of the number of atoms J is made with a criterion based on PCA [34]. PCA seeks to optimally represent the data in terms of the minimal reconstruction error, i.e., the mean-square-error between the representation and the original data, hence projecting onto the first eigenvectors of the covariance matrix of the inputs. We apply PCA to the data matrix, identifying the minimum number of eigenvectors necessary to explain at least $70 \%$ of the data covariance. We chose this value as the number of atoms J. Indeed, all of the state-of-the art tree inference methods $[27,35]$ have prohibitively long lists of single alterations, which is a very common scenario when dealing with complex diseases such as NB tumors.

The idea is to establish a mixture model $\mathrm{M}$ of $\mathrm{K}$ trees $\mathrm{T}_{k}$ with a maximum likelihood approach (22). In
MTreeMix, the estimation of a single tree is based on solving a maximum weight branching problem by a combinatorial algorithm. The mixture model is fitted iteratively with an EM algorithm: in the E-step, the algorithm assigns the data to the tree components and estimates the missing data; in the M-step, it fits the trees on the respective subsets. As input, MTreeMix considers the list of events or atom patterns, i.e., the lists of atoms used to reconstruct each sample. An event is an entry of a binary J-dimensional vector displaying which atoms are used by E-FLLat to reconstruct the corresponding sample (entries 0 and 1 denote the absence and the presence of an atom). To obtain the atom pattern, we considered a binarized version of the coefficient matrix $\Theta$, using a threshold that allowed us to discard the smallest noisy coefficients due to the inexactness of the minimization algorithm.

The algorithm then inferred a mixture $\mathrm{M}$ of trees $\mathrm{T}_{k}$, $M=\sum_{k=1}^{K} a_{k} T_{k}$, assuming that the data are generated by more than one stochastic process. Each tree of the mixture represents the probability distribution over the $2^{\mathrm{J}}$ possible patterns (with $\mathrm{J}$ atoms) associated with a set of events, and it is defined by vertices (random binary variables showing the occurrence of a single event) and weights (representing the conditional probability between events). The sum of weights $a_{k}$ associated with each tree sums to 1 , including the random mutations tree, a noisy tree associated with random mutations and represented by a star (all atoms belong to the first level and have a distance of one to the root node). Given a mixture of trees $\mathrm{M}$ and an atom pattern associated with a sample $\mathrm{x}$, one can estimate how well the mixture represents such a pattern by evaluating the likelihood of the pattern for each tree in the mixture: $L(x \mid M)=\sum_{k=1}^{K} L\left(x \mid T_{k}\right)$. The likelihood $L\left(x \mid T_{k}\right)$ indicates how likely it is that the sample $x$ belongs to the probability distribution defined by $T_{k}$. It must be noted that we used MTreeMix differently from the method for which it was originally proposed. In particular, the approach was designed for inferring the phylogenetic trees of single mutations. In other words, the algorithm can work on lists of single mutations. In our case, we used the same approach to infer a hierarchical structure (tree) of patterns of mutations, i.e., the atoms identified by E-FLLat. Our model indicates that stage 4 NB tumors show more complex CNA compared to the $4 \mathrm{~S}$ genetic tree. This is clearly indicated by the necessity to represent the oncogenic tree of stage 4 tumors using 42 atoms (Additional file 2 Figure S2). Additionally, GIP model requires two different oncogenic trees for stage 4 tumors compared to the one identified for stage $4 \mathrm{~S}$ tumors; in both cases, the level of noise (associated with the random mutations tree) is comparable ( 0.3 for stage $4 \mathrm{~S}$ and 0.37 for stage 4), However both stage 4 oncogenetic trees are marked diverged, up to five sublevels, explaining the greater 
variability and complexity of stage $4 \mathrm{NBs}$, also indicating their greater malignancy.

The increase of numerical CNAs observed in $4 \mathrm{~S}$ tumors, supports the chromosome endomitosis and abnormal mitosis as triggering events for malignant transformation. The endomitosis process [31] has been observed in a variety of physiological processes such as during the embryonic development, where the cells, as a nutriment and protection of the embryo, skip the cytokinesis step resulting in an increase of the ploidy [36]. The endomitosis process has been also described in cancer cells [37]. Kaneko and Knudson [38] reported the occurrence of endomitosis in stage $4 \mathrm{~S}$ cells. This process can generate aneuploid cells that undergoing to clonal expansion contribute to stage $4 \mathrm{~S}$ tumor development. Because the majority of $4 \mathrm{~S}$ tumors regress spontaneously, in the GIP model we may speculate that $4 \mathrm{~S}$ tumor cells, upon mitotic catastrophe, could undergo to apoptotic program to eliminate themselves, as initially proposed [39]. Another hypothesis already described in the stage $4 \mathrm{~S}$ regression [40] indicates that the same polyploidy cells could differentiate and then regress. The $4 \mathrm{~S}$ differentiation/regression process is further supported by our previous observation concerning upregulation of several genes belonging morphogenesis and differentiation, in particular the stage $4 \mathrm{~S}$ overexpressed genes implicated in peripheral nervous system development and in Ras-mediated cell death programs [8]. The endoreplication can further generate high DNA instability, rendering the genome prone to structural chromosome damage [41]. This situation leads to the coexistence, inside the tumor cells, of both numerical and structural CNAs, a condition often observed in stage 4 $[6,8]$. Contrarily to stage $4 S$, the stage 4 cells accumulate several genetic aberrations conferring proliferative

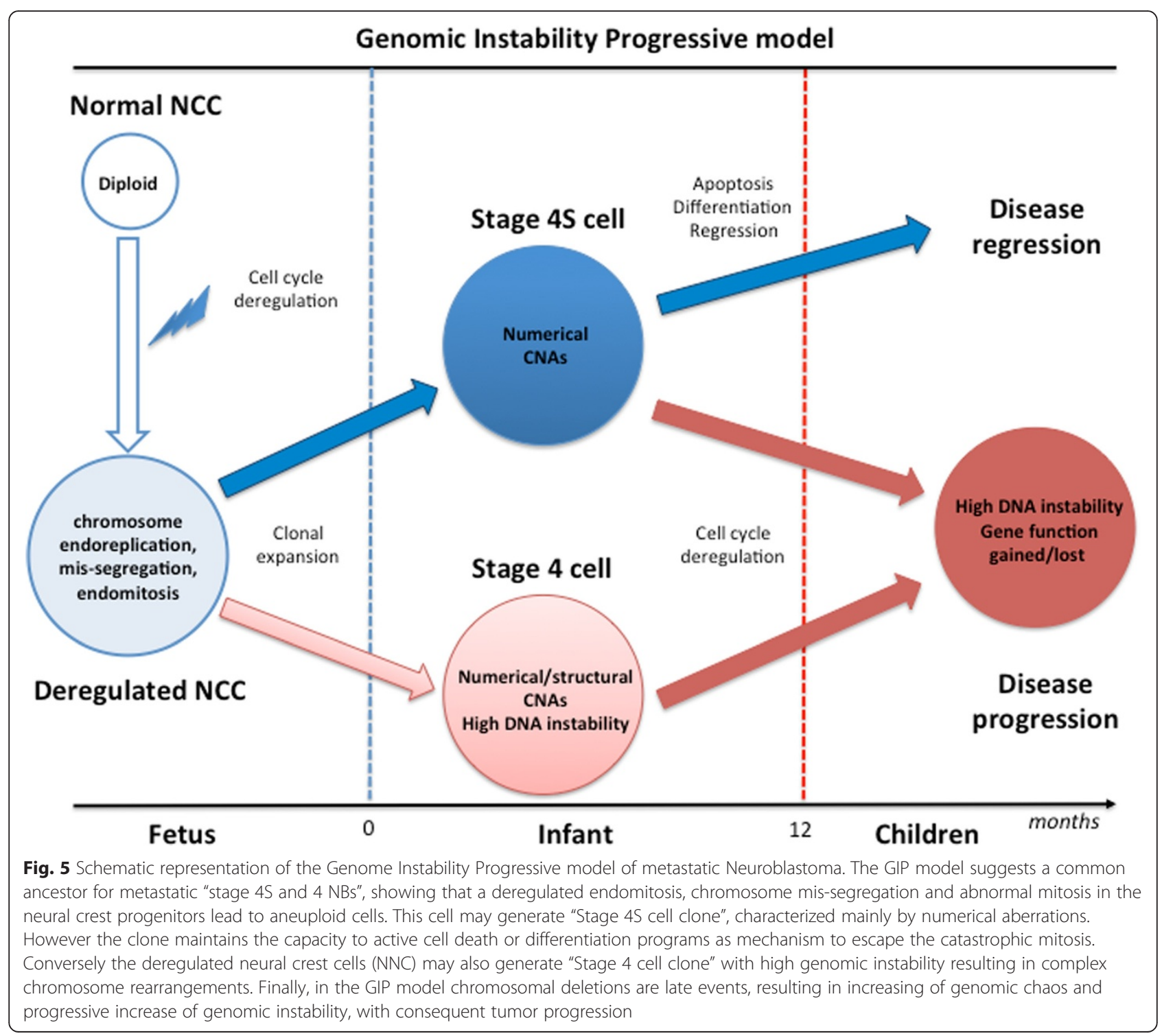


advantage and capacity to circumvent programmed cell death [6]. Additionally, since in this high aggressive tumor, the $17 \mathrm{q}$ gain is the most common aberrations as the initial GIP event, we could also hypothesize that this genomic imbalance represents the starting event that force to the tumorigenesis of stage 4 NBs.

The structural chromosome gain often results in a consequently overexpression of genes located within these regions. Specifically several genes, mapping in the long arm of chromosome 17, have been found overexpressed in NB. One of the most important gene within this region, is Survivin (BIRC5) (17q25), that encodes for an antiaptototic protein [42]. Survivin expression has been found markedly upregulated in neuroblastomas, and high level of expression also correlated with poor prognosis $[43,44]$. Similarity NME/NM23 nucleoside diphosphate kinase 1 (NME1) mapping in 17q21.3, has been also found overexpressed in some $\mathrm{NBs}$ and the upregulation correlated with metastatic disease [45].

It is interesting to note that in GIP model loss of chromosomes occur in the late event of carcinogenesis. In particular, stage $4 \mathrm{~S}$ tumors show chromosome deletion at different sub-levels of the atoms' tree (second A5, A6; third: A4, A9, A13 forth A8, A11). Notably, in stage 4 S genetic tree, only 1 out of 6 nodes displays diverse sublevels, with deletions mainly affecting the short arm of chromosome 14. Loss of 14q has been already involved in the NB initiation/progression [46] and may explain why a small portion of $4 \mathrm{~S}$ NBs develops toward a more aggressive disease.

On the other hand, the stage 4 tree shows loss at 11q that is not observed in stage $4 \mathrm{~S}$ tumors, and this deletion was present either in first (stage 4, left tree) and in the second level (stage 4, right tree). Interestingly in both stage 4 trees, 11q deletion is occurring before $3 p$ loss. This observation underlines the critical role of $11 \mathrm{q}$ deletion in stage 4 tumors suggesting that structural 11q aberration confers tumor aggressiveness as consequence of chromosome instability. Additionally, we also observe $1 p$ deletion at the secondary levels of stage 4 trees. Both these regions have been associated with an unfavorable clinical outcome and older patients [47]. All above data are in agreement with Kaneko and Knudson [38] that suggested how chromosome loss may occur after endoreplication and demonstrate that chromosome deletion is a late event linked to higher aggressiveness.

Differently from chromosome gain, the chromosome loss may produce breakage of gene sequence and dramatically lack of the gene function. Chromosome 1p36 deletion has been observed in approximately $36 \%$ of primary tumors and several studies indicate this region containing more than one NB suppressor genes including Tumor
Protein p73 (TP73) (1p36.32) [48], cyclin-dependent kinase 11B (CDK11B) (1p36.33) [49], neuroblastoma 1, DAN family BMP antagonist (NBL1) (1p36.13) [50] and paired box 7 (Pax7) (1p36.13) [51]. In addition to chromosome $1 \mathrm{p}$ other regions such as $3 \mathrm{p}, 11 \mathrm{q}$ and $14 \mathrm{q}$, frequently lost in NB, were also identified to contain putative NB suppressor genes. Specifically, RAS-association domain family 1 isoform $\mathrm{A}$ and (RASSF1A), mapping in 3p21.3, is a proapototic RAS effector and plays a key role in the DNA repair [52]. Cell adhesion molecule 1 (CADM1) gene, involved in the cell junction organization, was also reported as a good candidate NB suppressor gene that can be damaged by the deletion of the 11q23 region [53]. Lastly, chromosome 14q23 deletion, was observed in about $22 \%$ of NB and this locus contains MYC associated factor X (MAX) (14q23.3), that gives dimerization with $M Y C N$ gene [54], one of the most important oncogene associated with NB aggressiveness [55]. Lack of MAX repression function by chromosome 14q gene deletion may allow the overexpression of $M Y C N$ gene, as reported in aggressive NB. In conclusion, as suggested by GIP model, the occurring of chromosome 1p, 3p, 11q, and 14q deletions might increasing the aggressiveness of the tumor by damaging important NB suppressor genes.

Finally, because previous reports $[7,8]$ indicate that chromosome damages accumulates with increasing patient age, we evaluated the correlation between CNAs and patient age. We found a significant $(p=0.025)$ association between patient age and chromosome loss exclusively for stage 4 (atoms A15, A9; data not shown), supporting the hypothesis that $3 p$ and $11 \mathrm{q}$ chromosome deletion accumulates in older patients.

\section{Conclusions}

In conclusion, we propose for the first time a model of carcinogenesis for metastatic NB based on dictionary learning. Our model suggests that an aberrant regulation of the endomitosis could correlate to carcinogenesis process of metastatic NB. Afterwards, the polyploidy cells evolve in malignant clonal cell expansion generating stage $4 \mathrm{~S}$ or stage $4 \mathrm{NB}$, each of which is characterized by distinct genomic features. The former disease characterized by an increase of numerical chromosome aberrations, is able to activate a cell death or differentiation programs to escape the catastrophic mitotic. Conversely, stage 4 NBs show several complex chromosome rearrangements, where the chromosome deletions occur as late event, resulting in an increase of the genomic chaos and a progressive increase of chromosome instability, with consequent rapid disease progression (Fig. 5).

Future investigations of the NB carcinogenesis process can address future therapies to re-regulate the malignant cell cycle. 


\section{Endnotes \\ ${ }^{1}$ http://hgdownload.soe.ucsc.edu/goldenPath/hg19/ database/ \\ ${ }^{2}$ http://genome.ucsc.edu/cgi-bin/hgLiftOver.}

\section{Additional files}

Additional file 1: Figure S1. PCA analysis for stage 4S (top) and stage 4 (bottom) tumors. The vertical lines show the numbers of eigenvectors needed to explain $50 \%, 70 \%$ and $90 \%$ of the variance in the data. (TIFF $1811 \mathrm{~kb}$ )

Additional file 2: Figure S2. A plot of the stage 4 samples (top) and the corresponding reconstruction of the stage 4 sample data using $\mathrm{J}=42$ atoms (bottom). Red color indicates a gain, whereas blue corresponds to loss, as shown by the color bar. (TIFF $1521 \mathrm{~kb}$ )

\section{Abbreviations}

aCGH: Array comparative genomic hybridization; CNAs: Copy number aberrations; DL: Dictionary learning; E-FLLat: Enhanced fused lasso latent feature Model; GEO: Gene expression omnibus; GIP: Genome instability progressive; HG19: Human genome; NB: Neuroblastoma; PCA: Principal component analysis; QC: Quality control; TV: Total variation; UCSC: University of California, Santa Cruz (on-line genome browser).

\section{Competing interests}

The authors declare that they have no competing interest.

\section{Authors' contributions}

SM: planned the experimental design and performed the analyses; SC: participated in the design of the study, contributed to the data interpretation and wrote the manuscript; $A B$ : planned the experimental design and wrote the manuscript; AV: contributed to data interpretation and discussion; GPT: conceived of the study, contributed to results discussion and wrote the manuscript. All authors reviewed and approved the final manuscript.

\section{Acknowledgments}

The present work has been supported by Fondazione Italiana per la Lotta a Neuroblastoma. SC is a PhD supported by the Italian Ministry of Health (GR 2011-12; 02350922).

\section{Author details}

${ }^{1}$ DIBRIS, Università degli Studi di Genova, Genova, Italy. ${ }^{2}$ Lung Cancer Unit; IRCCS A.O.U. San Martino - IST, Genova, Italy. ${ }^{3}$ Neuroblastoma Laboratory, Onco/Hematology Laboratory, Department of Woman and Child Health, University of Padua, Pediatric Research Institute, Fondazione Città della Speranza, Padua, Corso Stati Uniti, 4, 35127 Padua, Italy.

\section{Received: 9 January 2015 Accepted: 28 August 2015}

\section{Published online: 10 September 2015}

\section{References}

1. Rubie H, De Bernardi B, Gerrard M, Canete A, Ladenstein R, Couturier J, et al. Excellent outcome with reduced treatment in infants with nonmetastatic and unresectable neuroblastoma without MYCN amplification: results of the prospective INES 99.1. J Clin Oncol. 2011;29:449-55.

2. Haupt R, Garaventa A, Gambini C, Parodi S, Cangemi G, Casale F, et al. Improved survival of children with neuroblastoma between 1979 and 2005: a report of the Italian Neuroblastoma Registry. J Clin Oncol. 2010;28:2331-8.

3. Scaruffi $P$, Coco S, Cifuentes F, Albino D, Nair M, Defferrari R, et al. Identification and characterization of DNA imbalances in neuroblastoma by high-resolution oligonucleotide array comparative genomic hybridization. Cancer Genet Cytogenet. 2007;177:20-9.

4. Oberthuer A, Berthold F, Warnat P, Hero B, Kahlert Y, Spitz R, et al. Customized oligonucleotide microarray gene expression-based classification of neuroblastoma patients outperforms current clinical risk stratification. J Clin Oncol. 2006;24:5070-8.
5. Schleiermacher G, Mosseri V, London WB, Maris JM, Brodeur GM, Attiyeh E, et al. Segmental chromosomal alterations have prognostic impact in neuroblastoma: a report from the INRG project. $\mathrm{Br} J$ Cancer. 2012;107:1418-22.

6. Stigliani S, Coco S, Moretti S, Oberthuer A, Fischer M, Theissen J, et al. High genomic instability predicts survival in metastatic high-risk neuroblastoma. In: Neoplasia (New York, NY), vol. 14. 2012. p. 823-32.

7. Schleiermacher $\mathrm{G}$, Janoueix-Lerosey I, Ribeiro A, Klijanienko J, Couturier J, Pierron $\mathrm{G}$, et al. Accumulation of segmental alterations determines progression in neuroblastoma. J Clin Oncol. 2010;28:3122-30.

8. Coco S, Theissen J, Scaruffi P, Stigliani S, Moretti S, Oberthuer A, et al. Agedependent accumulation of genomic aberrations and deregulation of cell cycle and telomerase genes in metastatic neuroblastoma. In: Int J Cancer, vol. 131. 2012. p. 1591-600.

9. Olshen $A B$, Venkatraman ES, Lucito $R$, Wigler M. Circular binary segmentation for the analysis of array-based DNA copy number data. In: Biostatistics, vol. 5. 2004. p. 557-72.

10. Willenbrock $\mathrm{H}$, Fridlyand J. A comparison study: applying segmentation to array CGH data for downstream analyses. In: Bioinformatics (Oxford, England), vol. 21. 2005. p. 4084-91.

11. Wang HJ, Hu J. Identification of Differential Aberrations in Multiple-Sample Array CGH Studies. In: Biometrics, vol. 67. 2010. p. 353-62.

12. Hupe P, Stransky N, Thiery JP, Radvanyi F, Barillot E. Analysis of array CGH data: from signal ratio to gain and loss of DNA regions. Bioinformatics. 2004;20:3413-22.

13. Pique-Regi R, Monso-Varona J, Ortega A, Seeger RC, Triche TJ, Asgharzadeh S. Sparse representation and Bayesian detection of genome copy number alterations from microarray data. Bioinformatics. 2008;24:309-18.

14. Tibshirani R, Wang P. Spatial smoothing and hot spot detection for $\mathrm{CGH}$ data using the fused lasso. Biostatistics. 2008;9:18-29.

15. Fridlyand J, Snijders AM, Pinkel D, Albertson DG, Jain AN. Hidden Markov models approach to the analysis of array CGH data. In: Journal of Multivariate Analysis, vol. 90. 2004. p. 132-53.

16. Price TS, Regan R, Mott R, Hedman A, Honey B, Daniels RJ, et al. SW-ARRAY: a dynamic programming solution for the identification of copy-number changes in genomic DNA using array comparative genome hybridization data. In: Nucleic Acids Res, vol. 33. 2005. p. 3455-64.

17. Lai WR, Johnson MD, Kucherlapati R, Park PJ. Comparative analysis of algorithms for identifying amplifications and deletions in array CGH data. In: Bioinformatics (Oxford, England), vol. 21. 2005. p. 3763-70

18. Pique-Regi R, Ortega A, Asgharzadeh S. Joint estimation of copy number variation and reference intensities on multiple DNA arrays using GADA. Bioinformatics. 2009:25:1223-30.

19. Mei TS, Salim A, Calza S, Seng KC, Seng CK, Pawitan Y. Identification of recurrent regions of Copy-Number Variants across multiple individuals. BMC Bioinf. 2010;11:147

20. Zhang Q, Ding L, Larson DE, Koboldt DC, McLellan MD, Chen K, et al. CMDS: a population-based method for identifying recurrent DNA copy number aberrations in cancer from high-resolution data. Bioinformatics. 2010;26:464-9.

21. de Ronde JJ, Klijn C, Velds A, Holstege H, Reinders MJ, Jonkers J, et al. KCSMARTR: An R package for detection of statistically significant aberrations in multi-experiment aCGH data. BMC Res Notes. 2010;3:298.

22. Picard F, Lebarbier E, Hoebeke M, Rigaill G, Thiam B, Robin S. Joint segmentation, calling, and normalization of multiple $\mathrm{CGH}$ profiles. Biostatistics. 2011:12:413-28.

23. Nowak G, Hastie T, Pollack JR, Tibshirani R. A fused lasso latent feature model for analyzing multi-sample aCGH data. In: Biostatistics, vol. 12. 2011. p. 776-91.

24. Vert J-P, Bleakley K. Fast detection of multiple change-points shared by many signals using group LARS. In: Advances in Neural Information Processing Systems 23, vol. 1. 2010. p. 1-9.

25. Tian Z, Zhang H, Kuang R. Sparse Group Selection on Fused Lasso Components for Identifying Group-specific DNA Copy Number Variations. In: Proc of IEEE ICDM. 2012.

26. Masecchia S, Barla A, Salzo S, Verri A. Dictionary learning improves subtyping of breast cancer aCGH data. Conf Proc IEEE Eng Med Biol Soc. 2013;2013:604-7.

27. Beerenwinkel N, Rahnenf "uhrer J, D\"aumer M, Hoffmann D, Kaiser R, Selbig J, et al. Learning multiple evolutionary pathways from cross-sectional data. In: Journal of Computational Biology, vol. 12. 2005. p. 584-98. 
28. Barrett T, Wilhite SE, Ledoux P, Evangelista C, Kim IF, Tomashevsky M, et al. NCBI GEO: archive for functional genomics data sets-update. In: Nucleic Acids Res, vol. 41. 2013. p. D991-995.

29. Jong K, Marchiori E, van der Vaart A, Chin S-F, Carvalho B, Tijssen M, et al. Cross-platform array comparative genomic hybridization meta-analysis separates hematopoietic and mesenchymal from epithelial tumors. In: Oncogene, vol. 26. 2007. p. 1499-506.

30. Meyer LR, Zweig AS, Hinrichs AS, Karolchik D, Kuhn RM, Wong M, et al. The UCSC Genome Browser database: extensions and updates 2013. In: Nucleic Acids Res, vol. 41. 2013. p. D64-69.

31. Van Houte BPP, Binsl TW, Hettling H, Pirovano W, Heringa J. CGHnormaliter: an iterative strategy to enhance normalization of array CGH data with imbalanced aberrations. In: BMC Genomics, vol. 10. 2009. p. 401.

32. Bleakley K, Vert J. The group fused lasso for multiple change-point detection. In: arXiv preprint. 2011.

33. Schwarz G. Estimating the dimension of a model. In: The Annals of Statistics. 1978.

34. Hastie T, Tibshirani RH, Friedman J. The elements of statistical learning: data mining, inference, and prediction. In: Book. 2009. p. 745.

35. Desper $\mathrm{R}$, Jiang F, Kallioniemi OP, Moch H, Papadimitriou CH, Schaffer AA. Inferring tree models for oncogenesis from comparative genome hybridization data. J Comput Biol. 1999;6:37-51.

36. Edgar BA, Orr-Weaver TL. Endoreplication cell cycles: more for less. Cell. 2001;105:297-306.

37. Storchova Z, Pellman D. From polyploidy to aneuploidy, genome instability and cancer. Nat Rev Mol Cell Biol. 2004:5:45-54.

38. Kaneko Y, Knudson AG. Mechanism and relevance of ploidy in neuroblastoma. In: Genes Chromosomes Cancer, vol. 29. 2000. p. 89-95.

39. Pritchard J, Hickman JA. Why does stage $4 \mathrm{~s}$ neuroblastoma regress spontaneously? Lancet. 1994;344:869-70.

40. Brodeur GM, Bagatell R. Mechanisms of neuroblastoma regression. Nat Rev Clin Oncol. 2014;11:704-13.

41. Shackney SE, Smith CA, Miller BW, Burholt DR, Murtha K, Giles HR, et al. Model for the genetic evolution of human solid tumors. Cancer Res. 1989:49:3344-54

42. Bown N, Cotterill S, Lastowska M, O'Neill S, Pearson AD, Plantaz D, et al. Gain of chromosome arm 17q and adverse outcome in patients with neuroblastoma. N Engl J Med. 1999;340:1954-61.

43. Islam A, Kageyama H, Takada N, Kawamoto T, Takayasu H, Isogai E, et al. High expression of Survivin, mapped to $17 q 25$, is significantly associated with poor prognostic factors and promotes cell survival in human neuroblastoma. Oncogene. 2000;19:617-23.

44. Islam A, Kageyama H, Hashizume K, Kaneko Y, Nakagawara A. Role of survivin, whose gene is mapped to 17q25, in human neuroblastoma and identification of a novel dominant-negative isoform, survivin-beta/2B. Med Pediatr Oncol. 2000;35:550-3.

45. Leone A, Seeger RC, Hong CM, Hu YY, Arboleda MJ, Brodeur GM, et al. Evidence for nm23 RNA overexpression, DNA amplification and mutation in aggressive childhood neuroblastomas. Oncogene. 1993;8:855-65.

46. Thompson PM, Seifried BA, Kyemba SK, Jensen SJ, Guo C, Maris JM, et al. Loss of heterozygosity for chromosome $14 \mathrm{q}$ in neuroblastoma. Med Pediatr Oncol. 2001;36:28-31.

47. Brodeur GM. Neuroblastoma: biological insights into a clinical enigma. Nat Rev Cancer. 2003;3:203-16.

48. Kaghad M, Bonnet H, Yang A, Creancier L, Biscan JC, Valent A, et al. Monoallelically expressed gene related to p53 at 1p36, a region frequently deleted in neuroblastoma and other human cancers. Cell. 1997;90:809-19.

49. Lahti JM, Valentine M, Xiang J, Jones B, Amann J, Grenet J, et al. Alterations in the PITSLRE protein kinase gene complex on chromosome 1p36 in childhood neuroblastoma. Nat Genet. 1994;7:370-5.

50. Enomoto H, Ozaki T, Takahashi E, Nomura N, Tabata S, Takahashi H, et al. Identification of human DAN gene, mapping to the putative neuroblastoma tumor suppressor locus. Oncogene. 1994;9:2785-91.

51. Shapiro DN, Sublett JE, Li B, Valentine MB, Morris SW, Noll M. The gene for PAX7, a member of the paired-box-containing genes, is localized on human chromosome arm 1p36. Genomics. 1993;17:767-9.

52. Yang Q, Zage P, Kagan D, Tian Y, Seshadri R, Salwen HR, et al. Association of epigenetic inactivation of RASSF1A with poor outcome in human neuroblastoma. Clin Cancer Res. 2004;10:8493-500.
53. Michels E, Hoebeeck J, De Preter K, Schramm A, Brichard B, De Paepe A, et al. CADM1 is a strong neuroblastoma candidate gene that maps within a 3.72 Mb critical region of loss on 11q23. BMC Cancer. 2008;8:173.

54. Cascon A, Robledo M. MAX and MYC: a heritable breakup. Cancer Res. 2012;72:3119-24.

55. Tonini GP, Pistoia V. Molecularly guided therapy of neuroblastoma: a review of different approaches. Curr Pharm Des. 2006;12:2303-17.

\section{Submit your next manuscript to BioMed Central and take full advantage of:}

- Convenient online submission

- Thorough peer review

- No space constraints or color figure charges

- Immediate publication on acceptance

- Inclusion in PubMed, CAS, Scopus and Google Scholar

- Research which is freely available for redistribution

Submit your manuscript at www.biomedcentral.com/submit 